\title{
Effects of Connection Type between Surface Vessel and Submersible Propeller on Motion Performance of Wave Glider
}

\author{
Zhanxia Feng ${ }^{1,2}$, Zongyu Chang ${ }^{1,2,3, *}$, Zhongqiang Zheng ${ }^{1,2}$ and Panfeng Wang ${ }^{2,4}$ \\ 1 College of Engineering, Ocean University of China, Qingdao 266100, China; 15063008682@163.com (Z.F.); \\ zqzheng@ouc.edu.cn (Z.Z.) \\ 2 Key Laboratory of Mechanism Theory and Equipment Design of Ministry of Education, Tianjin University, \\ Tianjin 300072, China; panfengwang@tju.edu.cn \\ 3 Key Lab of Ocean Engineering of Shandong Province, Qingdao 266100, China \\ 4 School of Mechanical Engineering, Tianjin University, Tianjin 300072, China \\ * Correspondence: zongyuchang@ouc.edu.cn; Tel.: +86-132-8085-8045
}

Received: 7 November 2018; Accepted: 22 November 2018; Published: 3 December 2018

\begin{abstract}
Wave Glider is an autonomous surface vehicle that directly uses wave energy to generate forward power and has been widely used in marine survey and observation. Wave Glider is composed of surface vessel, submersible propeller and the connection structure between them. Connection types are thought to be related to the performance of Wave Glider closely. In this paper, the effects of the connection structure between the surface vessel and the submersible propeller on the motion performance of the Wave Glider are studied. Several connection types such as rigid rod, cable, multi-link chain and elastic rod are applied to connect the surface vessel and the submersible propeller. The models of connection structures are developed respectively. Among them, cable model is established with a finite number of small cylinders, which connected by spring and damping elements; multi-link chain can be seen as hinged by multiple rigid rods; elastic rod model can be looked on as several segments linked with elastic components. Considering the connection characteristics, the integrated dynamic models are established by applying multi-body dynamics software ADAMS (Automatic Dynamic Analysis of Mechanical Systems) with consideration of the hydrodynamic forces on different components of Wave Glider. The propulsion performance of the Wave Glider is calculated by using numerical method, and the simulation results showed that the difference of propulsion performance with different connection types of the Wave Glider is slightly. But serious impacts can occur on the connections of rigid rod and multi-link chain. They can lead to serious extra load on the structure of Wave Glider. From the engineering practice of Wave Glider application, the cable connection is more convenient to transport, deploy, recover and store. It is also the generous connection type for wave glider.
\end{abstract}

Keywords: Wave Glider; connection structure; dynamic performance; wave energy utilization

\section{Introduction}

As an ocean mobile observation platform, Wave Glider has been widely used in marine resources development, maritime exploration and military fields [1-3].

Many researchers studied the equipment from different aspects. On design and optimization of Wave Glider, Li et al. [4,5] conducted a preliminary study of whole performance of the Wave Glider, provided the design basis for the length and width of the glider and the length of the connecting cable, and realize the modeling and analyzing of Wave Glider by using the co-simulation of ADAMS and MATLAB software; Wang et al. [6] proposed an optimization method based on the Latin Hypercube 
Sampling (LHS) method and Computational Fluid Dynamics (CFD) method for the submersible propeller which is Blended Wing Body (BWB) layout, and obtained an optimization model for the BWB Water Glider; Tian et al. [7] introduced the flexible webbed wings based on the researches of traditional Wave Glider, conducted an intensive study on the propulsion principle of the Wave Glider with webbed wings (WGWW), established a driving force calculation model of the flexible webbed wing and realized the glide function of the WGWW; J. Rice et al. [8] designed and analyzed a recovery system to improve Wave Glider, the proposed solution was demonstrated to be feasible and met all design requirements, with an emphasis on simplicity.

In order to study the kinematic performance of the Wave Glider, Alaaeldeen M. E. Ahmed et al. [9], using computational fluid dynamics (CFD) to predict calm water resistance and seakeeping for the floating hull of Wave Glider, and concluded that the NPL hull is the optimum hull compared to the other hulls; Ryan N. Smith et al. [10] determined the kinematics model for the Wave Glider platform developed by Liquid Robotics and estimated the speed and environmental parameters for a desired heading; Phillip Ngo et al. [11] applied Gaussian process models to existing wave model data to predict the performance, and carried out an effective method for forecasting WG velocity; Liu [12] analyzed the motion of a Wave Glider and studied its forward speed at different sea states based on CFD numerical simulation technology.

For determining the effect of the connection cable on the performance of Wave Glider, Zhou et al. [13] established a dynamic model of the Wave Glider by using the Kane method, and analyzed the effect of sea states and cable lengths on the propulsion performance. Zhang et al. [14] discretized the cable that connected the surface vessel and the submersible propeller into several small segments for analysis, and obtained the motion equations between the surface vessel and the submersible propeller, and found that the speed of the Wave Glider is easy to adjust as the wave changes due to the effect of the flexible cable, in addition, the short cable is in favor of motion transmission. Liquid Robotics proposed two kinds of design, one is a rigid hinged rod consisting of two hinged rigid rods, which can improve operability for deployment and recycling of the Wave Glider, another has two independent rigid rods, which is applied to reduce the possibility of cable twisting [15].

The connection structure is an important component for the safety and reliability of the Wave Glider. It is essential to study the effects of the connection types on the performance of the Wave Glider. In this paper, the relationship between connection structure and the performance of the Wave Glider is studied. Several connection types such as rigid rod, cable, multi-link and elastic rod are considered to connect the surface vessel and the submersible propeller. Considering the connection characteristics, the integrated dynamic models are established by applying multi-body dynamics software ADAMS with consideration of the hydrodynamic forces on different components of Wave Glider, and the horizontal displacement and velocity of the surface vessel and the tension forces on the connection structure with different types have been compared.

\section{Materials and Methods}

\subsection{Types and Models of Connection Components}

The Wave Glider consists of surface vessel, connection structure and submersible propeller. With the excitation of wave, the surface vessel moves in a heave direction. Then the wings rotate under the action of hydrodynamic force induced heave and the thrust generates and pushes the vessel forward.

To study the effects of cable connection method on motion performance of Wave Glider, a dynamic model is established. In order to simplify the calculation, the two-dimensional small-amplitude wave is taken as the basis for dynamic simulation of Wave Glider, and the following assumptions are proposed:

1. The Wave Glider does not change the forward direction during the movement and moves only in the two-dimensional plane;

2. The shape of the surface vessel has no effects on the motion performance of the Wave Glider; 
3. Only heave motion is considered of surface vessel and the heave motion is defined as follows:

$$
\eta=A \cos (k x-\omega t)
$$

where $\eta$ is the motion equation of the surface vessel in vertical direction; $A$ is the wave amplitude; $k x$ is the phase; $\omega$ is the angular frequency

4. The hydrodynamic force of the surface vessel and the wings are given based on the Morison equation:

$$
f_{D}=\frac{1}{2} C_{D} \rho A u_{x}\left|u_{x}\right|
$$

where $C_{D}$ is the drag coefficient, $\rho$ is the density of the fluid, $A$ is the projected area of a unit length of the surface vessel perpendicular to the flow direction, and $u_{x}$ is the horizontal velocity.

Four types of connection models of rigid rod, cable, multi-link and elastic rod are considered to analyze the propulsion performance of the Wave Glider, which are shown as Table 1.

Table 1. Model of four connection types.

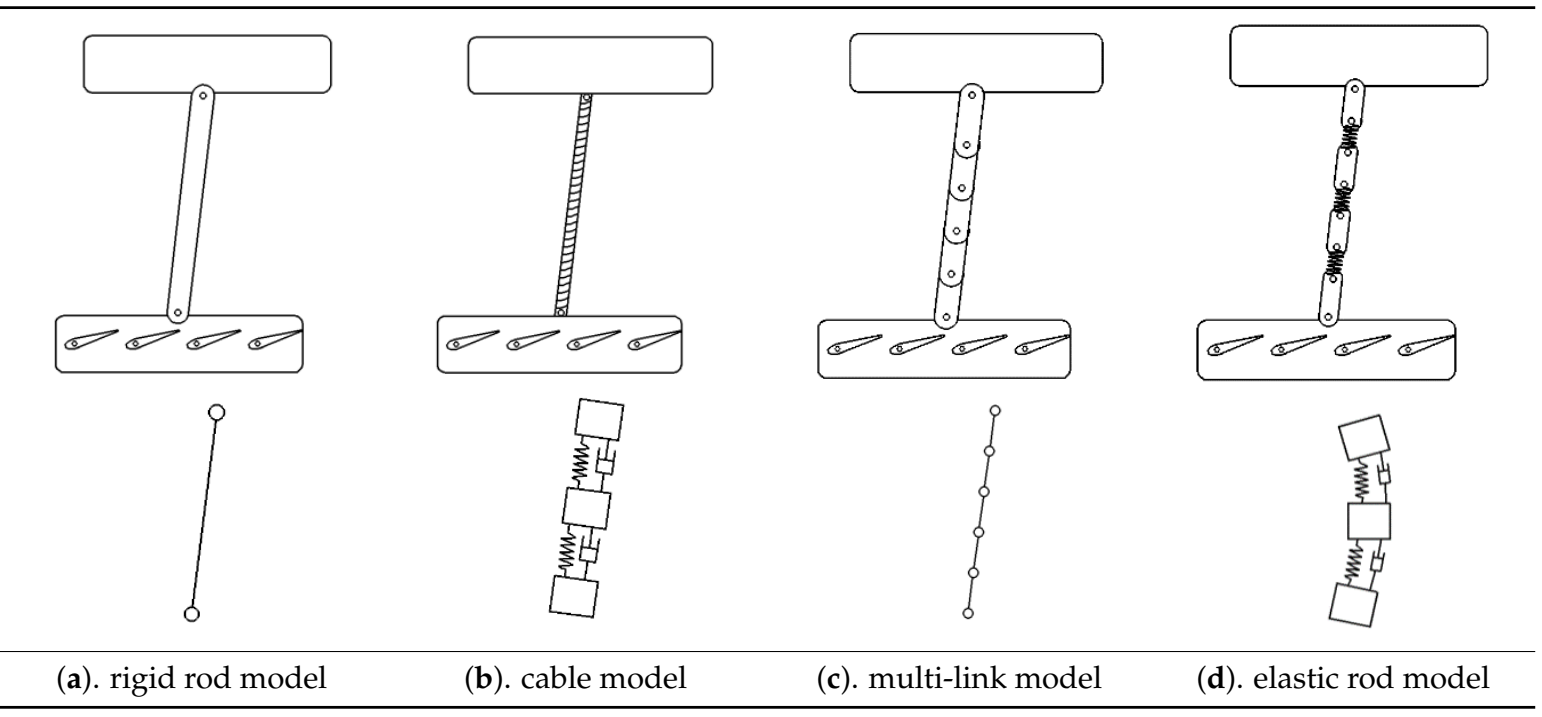

The rigid rod can be seen as a link connected the floating body and propeller; the cable is established with a finite number of small cylinders, which connected by spring and damping forces; the multi-link can be regarded as links connected by hinges; for the connection elastic rod, it can be looked on as several segments linked with massless elastic components.

\subsection{Multibody Dynamics Modeling of Wave Glider with Different kind of Connection}

For the ADAMS dynamic modeling and simulation of Wave Glider, the propulsion performance of the Wave Glider under different conditions was obtained by numerically solving the dynamic equation considering the fluid-structure interaction and rigid-flexible coupling. The model used in this study was firstly established in 3D software SolidWorks and then imported into ADAMS. The model is shown in Figure 1. 


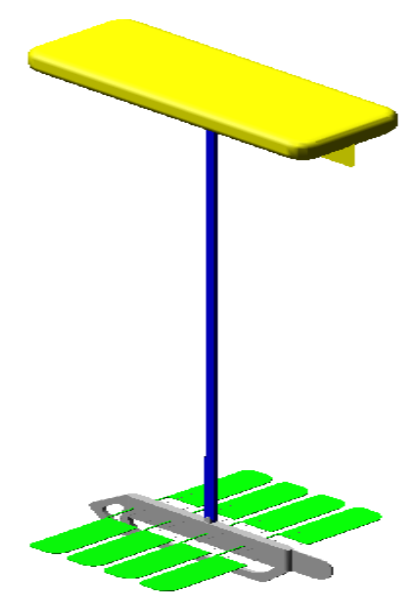

Figure 1. The 3D model of the Wave Glider.

The parameters of the Wave Glider are provided in Table 2.

Table 2. The parameters of the Wave Gliders.

\begin{tabular}{cc}
\hline Parameters & Value \\
\hline Length of surface vessel/m & 1.4 \\
Width of surface vessel/m & 0.52 \\
Height of surface vessel/m & 0.15 \\
Number of wings/pair & 4 \\
Area of single wing/m $\mathrm{m}^{2}$ & 0.06 \\
Length of cable/m & 3 \\
\hline
\end{tabular}

After the model is imported into ADAMS, the cable is re-modeled and the four models of rigid rod, cable, multi-link and elastic rod are respectively built.

In the re-modeling process, the attributes, constraints, forces, and movements of the model need to be defined and added. The connection structure and the surface vessel and the submersible propeller are connected with revolute joints; the revolute joints are added at the rotating shaft of the wings of the submersible propeller, and the contact force is added between the wings and the position limiting device; the surface vessel and the wings are subjected to the hydrodynamic force during movement, and according to the Morrison equation, the corresponding wave force is added; the surface vessel is added with a simple harmonic motion to simulate the heaving motion of the waves.

\section{Results and Discussion}

After the modeling of the Wave Glider with different connection types, the movement simulation and analysis are performed and the simulation results are as follows.

\subsection{Displacement Analysis of the Surface Vessel}

Through the comparison of the displacements of the surface vessel in the four connection types as shown in Figure 2, it can be seen that the displacement of the cable is larger, and the displacement curves of the surface vessel of the other three connection methods are almost coincident, as a result, the displacement characteristics of the Wave Glider using the cable connection method are better. 


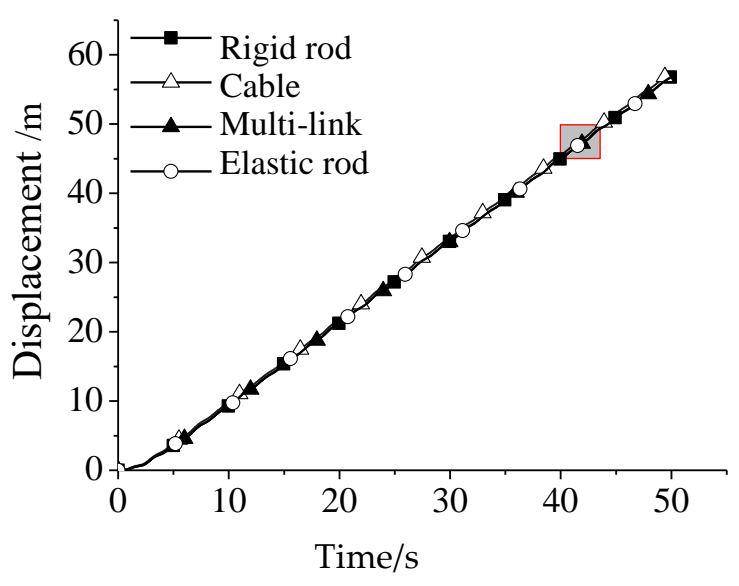

(a)

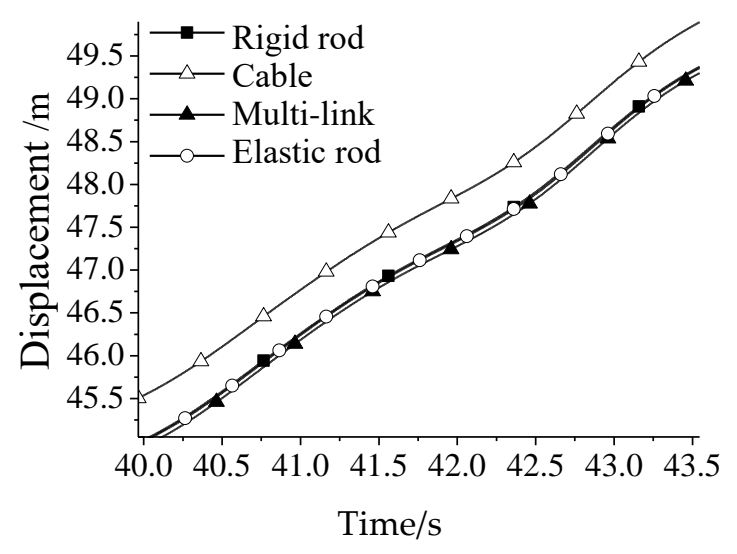

(b)

Figure 2. Displacements of surface vessel in four connection types. (a) Displacement response. (b) Enlarge displacement response curves.

\subsection{Velocity Analysis of the Surface Vessel}

Due to the regular harmonic motion of the surface vessel along with the waves, the velocity of the surface vessel also fluctuates regularly as shown in Figure 3. It can be seen from the comparison of the surface vessel velocity in the four connection types that the fluctuation of the forward speed of four connection methods is consistent, and they have little difference in the effect on the velocity of the surface vessel, the time when the velocity reaches a stable level is approximately the same. The multi-link and the elastic rod have the highest maximum horizontal velocity, which is about $1.6 \mathrm{~m} / \mathrm{s}$, the maximum horizontal velocity of the cable and the rigid rod are about $1.58 \mathrm{~m} / \mathrm{s}$. From the mean velocity as shown in Figure 4, it can be seen that the mean velocity of the four connection methods is not much differently, the mean velocity of the cable is slightly larger. However, on the whole, the maximum velocity of the four types of connection is not significantly different, and the surface vessel with cable has smaller velocity fluctuation and better performance.

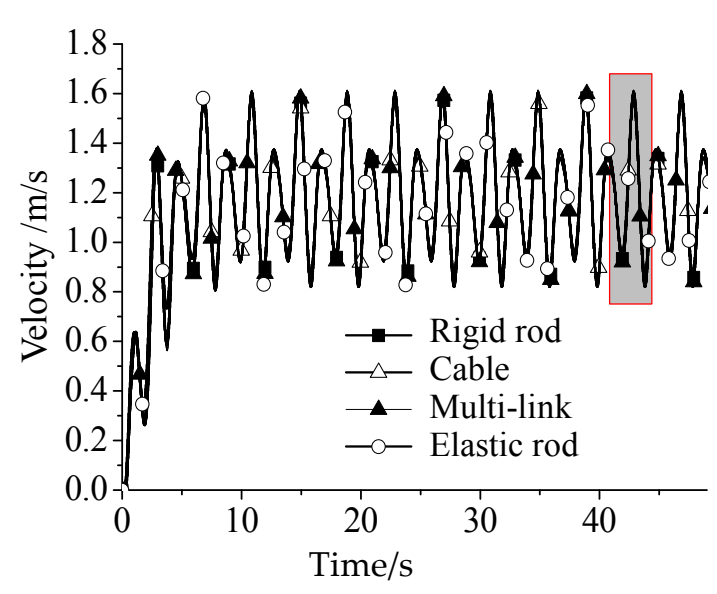

(a)

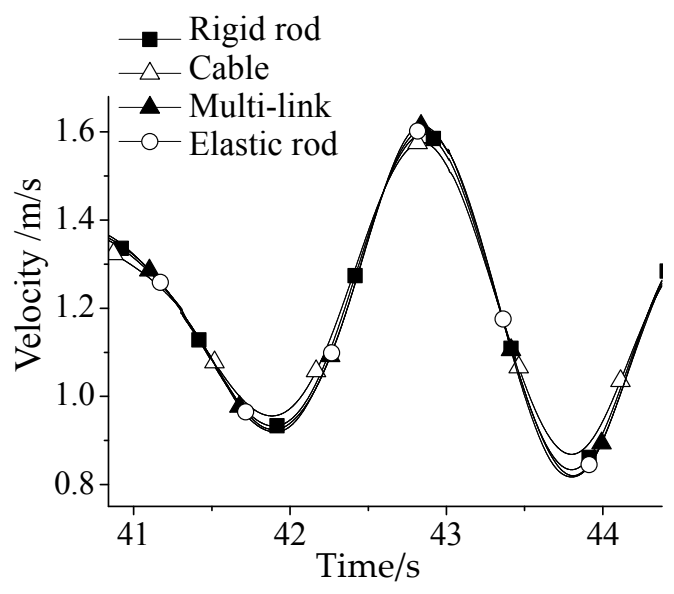

(b)

Figure 3. Velocity of surface vessel in four connection types. (a) Velocity response. (b) Enlarge velocity response curves. 


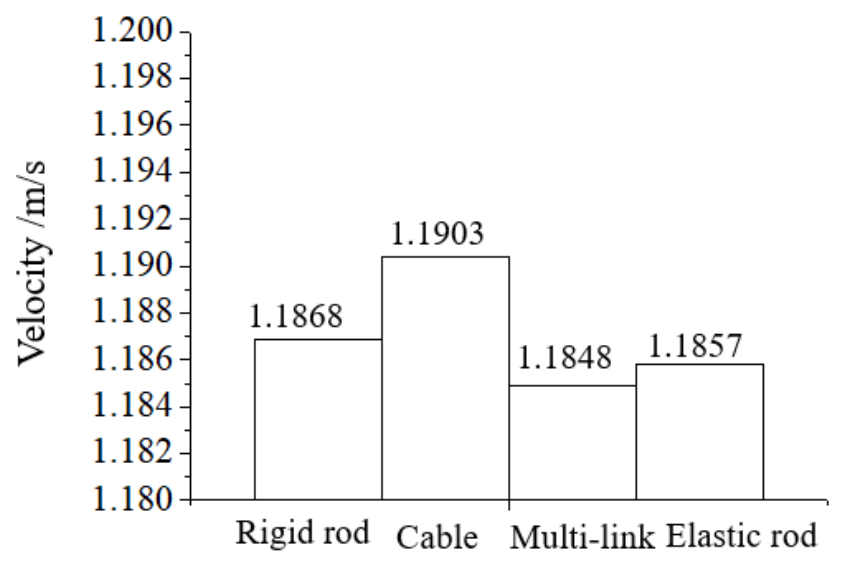

Figure 4. Mean velocity of surface vessel in four connection types.

\subsection{Force Analysis of the Hinged Point between the Connection Structure and the Surface Vessel}

The propulsion of the wave glider is mainly generated by the submersible propeller. The surface vessel of the water moves up and down with the undulating motion of the waves, and the motion of the surface vessel is transmitted to the submersible propeller through the connection structure, therefore, the submersible propeller performs the heave motion, and the wings of the submersible propeller are deflected by the force of the water, generating forward propulsion. Propulsion is concentrated at the hinge point, which is the pull force relative to the surface vessel. It can be seen from the horizontal pull force curves of the hinged point of four connection types that the four pull force curves almost coincide with each other, which shows that the connection types have little effect on the pull force of the hinged point, but due to the momentary contact and collision between the wings and the position limiting device, the pull force of the hinged point is affected and suddenly serious impacts occurs, as shown in Figure 5. It can be seen from all the pull force curves of different connection types that the pull force of the hinged point of the rigid rod and the multi-link all have serious impacts, and due to the elastic rod and the cable have a certain damping effect, the sudden impacts on the pull force of the hinged point is small. Overall, the cable has the smallest sudden impacts and the best dynamic performance.

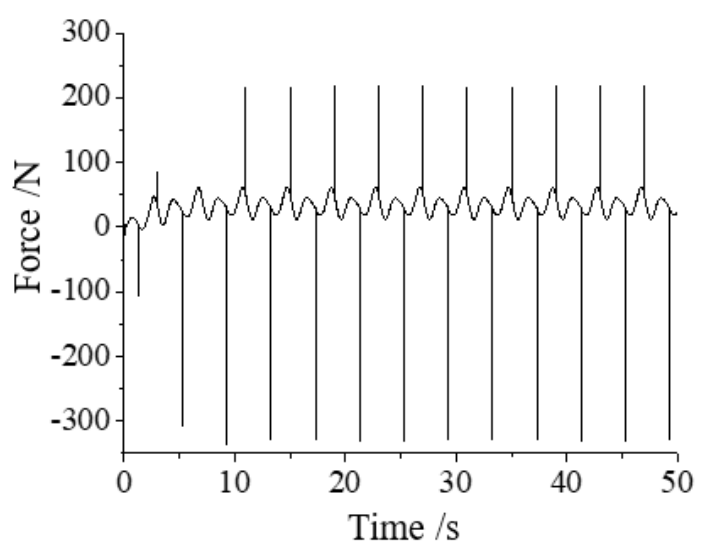

(a)

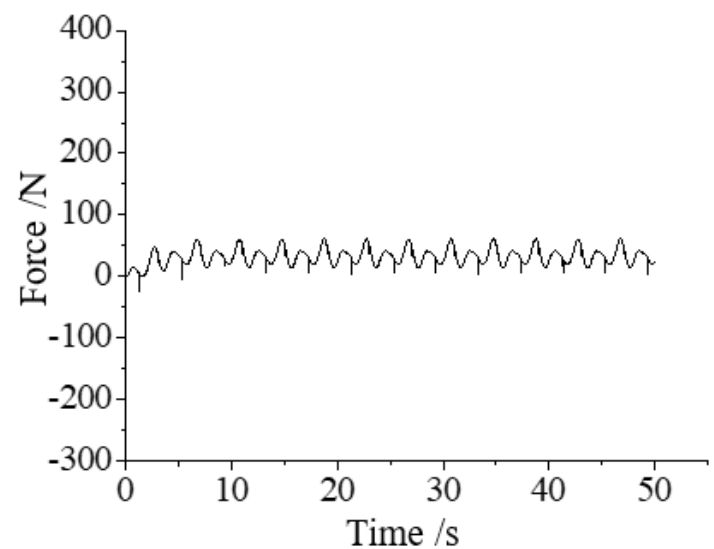

(b)

Figure 5. Cont. 


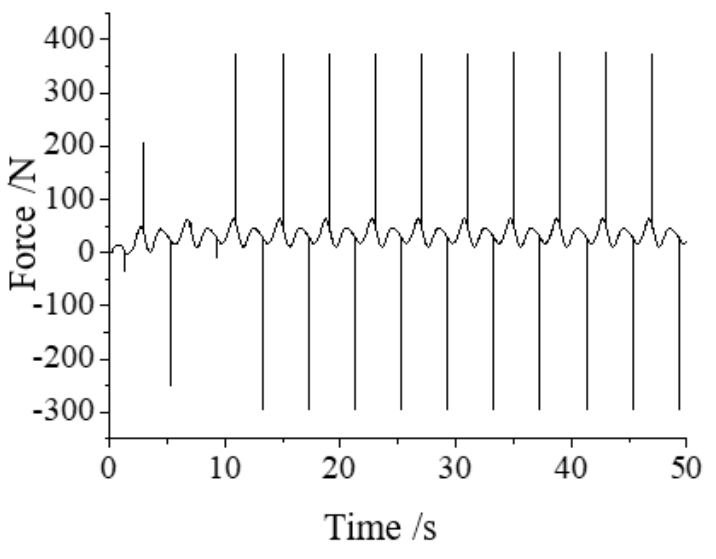

(c)

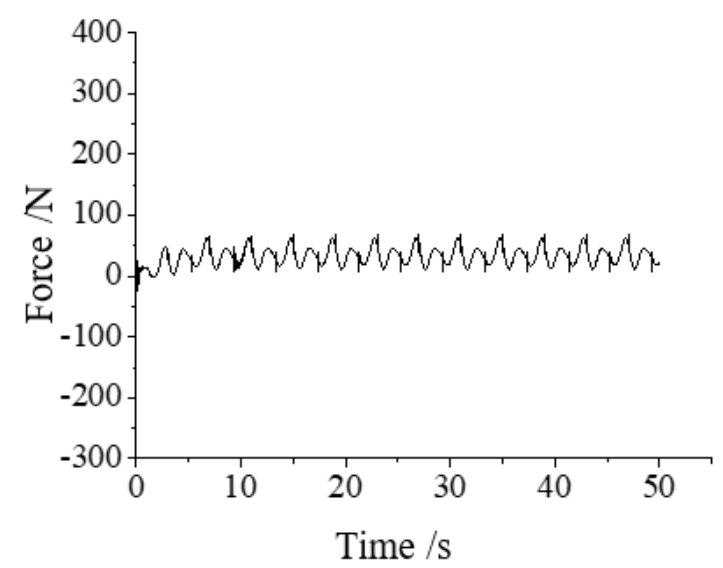

(d)

Figure 5. Comparison of horizontal pull force of the hinged point of four connection types. (a) Horizontal pull force of the hinged point with rigid rod; (b) Horizontal pull force of the hinged point with cable; (c) Horizontal pull force of the hinged point with multi-link; (d) Horizontal pull force of the hinged point with elastic rod.

\section{Conclusions}

In this paper, the dynamics modeling and simulation of the Wave Glider with different connection types were studied. The effect of the different connection types on the dynamic performance of the Wave Glider were analyzed by using the multi-body dynamics software ADAMS. According to dynamic response, the following conclusions were drawn:

1. When the Wave Glider respectively connected by rigid rod, multi-link and elastic rod, the displacement responses are similar and the difference is not obvious among different connection types. The motion of vessel with the cable is slight fast.

2. The velocity responses of the four types of connection structures are also similar; the mean velocity of the Wave Glider with cable connection is faster than rigid rod, elastic rod and multi-link. And the vessel with cable also has smaller fluctuation speed.

3. The connection types have little effect on the horizontal pull force of the hinged point between the connection structure and the surface vessel. Serious impacts can suddenly occur with connection of rigid rod and multi-link, which will lead to serious extra load on the structure of Wave Glider. Due to the elastic rod and the connection cable have a certain damping effect, the sudden impacts on the pull force of the hinged point is small. Overall, the cable connection has the smallest suddenly impacts of the pull force and the best performance.

Author Contributions: Conceptualization, Z.C.; Investigation, P.W.; Methodology, Z.F., Z.C. and Z.Z.; Resources, P.W.; Software, Z.F. and Z.Z.; Supervision, Z.C., Z.Z. and P.W.; Writing—original draft, Z.F.; Writing一review \& editing, Z.F. and Z.C.

Funding: This research was funded by the National Natural Science Foundation of China, grant number 51875540; The Fundamental Research Funds for the Central Universities, grant number 201513053.

Acknowledgments: The authors are grateful for the financial support of National Natural Science Foundation of China (No. ), The Fundamental Research Funds for the Central Universities (No. ) and Key Laboratory of Mechanism Theory and Equipment, Design of Ministry of Education (No. )

Conflicts of Interest: The authors declare no conflict of interest.

\section{References}

1. Hu, M.Z.; Liu, Q.B.; Xiao, Z.J.; Wang, Z.L.; Zhao, Y.H. Design and Experimental Test for Principle Prototype of Wave Glider. Mine Warf and Ship Self-Def. 2017, 25, 16-19. 
2. Manley, J.; Willcox, S. The Wave Glider: A new concept for deploying ocean instrumentation. IEEE Instrum. Meas. Mag. 2010, 13, 8-13. [CrossRef]

3. Coxsworth, B. Liquid Robotics Announces Next Generation of Wave Glider Sea-Going Robot; New Atlas: Tulsa, OK, USA, 2013.

4. Li, X.T.; Wang, L.; Wu, X.T.; She, H.Q. Principle and System Design of a Wave Glider. J. Sichuan Ordnance 2013, 34, 128-131.

5. Li, X.T. Dynamic Model and Simulation Study Based on the Wave Glider; China Ship Research and Development Academy: Beijing, China, 2014.

6. Wang, Z.Y.; Wang, Y.X.; Yu, J.C.; Zhang, A.Q. Hydrodynamic Shape Optimization for the Flying-Wing Underwater Glider Based on Improved LHS Method. J. Ocean Technol. 2017, 36, 50-56.

7. Tian, B.Q.; Li, L.L. Structure Design and Movement Principle of Wave Gliders with Webbed Wings. China Mech. Eng. 2017, 28, 2939-2943.

8. Rice, J.; Gish, L.A.; Barney, J.; Gawboy, Z.; Mays, B.; Moore, L.; Nickell, A. Design and analysis of an improved Wave Glider recovery system. In Proceedings of the OCEANS 2016 MTS/IEEE Monterey, Monterey, CA, USA, 19-23 September 2016.

9. Ahmed, A.M.E.; Duan, W. Resistance and Seakeeping Investigation for Optimization of the Floating Hull of Wave Glider. World J. Eng. Technol. 2016, 4, 235-249. [CrossRef]

10. Smith, R.; Das, J.; Hine, G.; Anderson, W.; Sukhatme, G.S. Predicting Wave Glider speed from environmental measurements. In Proceedings of the OCEANS'11 MTS/IEEE KONA, Waikoloa, HI, USA, 19-22 September 2011.

11. Ngo, P.; Das, J.; Ogle, J.; Thomas, J.; Anderson, W.; Smith, R.N. Predicting the speed of a Wave Glider autonomous surface vehicle from wave model data. In Proceedings of the 2014 IEEE/RSJ International Conference on Intelligent Robots and Systems, Chicago, IL, USA, 14-18 September 2014; pp. 2250-2256.

12. Liu, P.; Zhang, H. Speed Prediction of Wave Glider based on CFD Method. Ship Sci. Technol. 2016, 38, $108-111$.

13. Zhou, C.L.; Wang, B.X.; Zhou, H.X.; Li, J.L.; Xiong, R. Dynamic Modeling of a Wave Glider. Front. Inf. Technol. Electron. Eng. 2017, 18, 1295-1304. [CrossRef]

14. Zhang, C.Y.; Xu, X.S. Research on Motion Relationship Between the Surface Boat and the Wave-Propelled Sub in Wave Glider System. J. Sichuan Ordnance 2018, 52, 127-132.

15. Hine, R.G. Wave-Powered Device with One or More Tethers Having One or More Rigid Sections. WO 2012126012, 28 February 2013. 